\title{
La relación entre la ingesta de calcio y los eventos cardiovasculares no es concluyente
}

The association between calcium intake and cardiovascular events is inconclusive

Li K et al. Heart. 2012;98(12):920-5

\section{Objetivos}

Evaluar la asociación entre la ingesta de calcio (de la dieta y/o suplementos) con el riesgo de infarto agudo de miocardio (IAM), accidente cerebrovascular (ACV) y mortalidad por enfermedades cardiovasculares (ECV).

\section{Diseño, lugar y pacientes}

Cohorte prospectiva (Cohorte Europea de Cáncer y Nutrición, EPIC). Residentes de Heidelberg, Alemania, de entre 35 y 64 años de edad libres de cualquier ECV al momento del reclutamiento (realizado entre 1994 y 1998). Fueron excluidos aquellos cuya ingesta energética diaria estuviera en los extremos (percentil 0,5 superior o inferior).

Medición de Resultados Principales

Incidencia de IAM, ACV y mortalidad por ECV.

\section{Resultados Principales}

A mayor ingesta de calcio de la dieta se observó: menor edad, mayor nivel educativo, mayor actividad física, menor probabilidad de ser fumador, tener sobrepeso u obesidad, y menor consumo de alcohol. La ingesta de calcio de la dieta también se asoció positivamente con la ingesta de vitamina $D$, ácidos grasos saturados, ingesta total de proteínas y probabilidad de tomar suplementos de calcio.

En comparación con los no usuarios, los usuarios de los suplementos de calcio fueron en su mayoría mujeres, físicamente más activos y menos propensos a sobrepeso $u$ obesidad. Los usuarios de suplementos de calcio fueron de edad más avanzada, nivel educativo más bajo e historial más prolongado de tabaquismo.

Los principales resultados se resumen en la tabla 1. No se observó asociación estadísticamente significativa entre la ingesta de calcio de la dieta o en forma de suplementos y la incidencia de ACV o la mortalidad por ECV.

Tabla1: asociación entre ingesta total de calcio en la dieta o suplementos y eventos cardiovasculares.

\begin{tabular}{l|c|c|c|c}
\multirow{2}{*}{\multicolumn{1}{c|}{ Resultado }} & \multicolumn{2}{|c|}{ Ingesta de calcio de la dieta } & Ingesta de suplementos de calcio \\
\cline { 2 - 5 } & Cuartil & HR (IC 95\%) ${ }^{\alpha}$ & Ninguno de suplemento & HR (IC 95\%) \\
\hline Incidencia de infarto & 1 (bajo) & 1 (ref) & Calcio & 1 (ref) \\
agudo de miocardio & 2 & $0,94(0,70$ a 1,25$)$ & Calcio solo & $1,86(1,17$ a 2,96$)$ \\
& 3 & $0,69(0,50$ a 0,94$)$ & Calcio + otros & $2,39(1,12$ a 5,12$)$ \\
& 4 (alto) & $0,85(0,63$ a 1,16$)$ & Otros suplementos & $1,66(0,95$ a 2,93$)$ \\
& $p$ & 0,22 & $0,75(0,58$ a 0,97$)$ \\
\hline
\end{tabular}

HR: Hazard ratio. a: ajustado por sexo, edad, nivel educativo, actividad física, BMI, tabaquismo, consumo de alcohol, ingesta de vitamina D, ingesta de proteínas y grasas saturadas, ingesta calórica total, diabetes mellitus.

\section{Conclusiones}

El incremento en la ingesta de calcio de la dieta no proveería beneficios significativos a nivel cardiovascular, mientras que el uso de suplementos de calcio podría incrementar el riesgo de
IAM y deben utilizarse con precaución.

Fuente de financiamiento: Deutsche Krebshilfe y Graduiertenkolleg. Conflicto de interés de los autores: ninguno reportado.

\section{Comentario}

La diferencia de ingesta de calcio proveniente de lácteos como de suplementos en la población argentina comparada con la que presenta el estudio hace difícil que las conclusiones sean apropiadas para su aplicación en nuestra población. Los datos de este estudio reportan una ingesta media entre 766 y $828 \mathrm{mg}$ mientras que los datos disponibles en Argentina muestran una ingesta mucho más baja'. El tipo de estudio usado tiene limitaciones propias de los estudios no aleatorizados que impiden determinar asociaciones fuertes y sacar conclusiones definitivas, a pesar que los resultados sean ajustados por confundidores ${ }^{2}$. El estudio remarca que los grupos con mayor y menor ingesta de calcio proveniente de lácteos o de suplementos eran significativamente diferentes. Si bien se halló una asociación significativa entre uso de suplementos de calcio e incremento de riesgo de IAM, este resultado podría estar sesgado por la presencia de otros confundidores asociados al uso de suple- mentos de calcio ${ }^{3}$. Por otro lado la ingesta de calcio fue evaluada en la población al momento del reclutamiento y en un seguimiento a los dos o cuatro años de reclutamiento mientras que los eventos fueron medidos a lo largo de los 11 años del estudio. Asimismo las dosis de suplementos no fueron registradas. Estudios aleatorizados controlados con placebo tampoco fueron concluyentes con los resultados del aumento de riesgo de infarto de miocardio $4,5,6,7,8,9$. Sería interesante que estos estudios muestren si los riesgos fueron diferentes dependiendo de la cantidad de calcio total consumida ${ }^{10}$.

\section{Conclusiones del comentador}

La ingesta de suplementos de calcio en dosis que excedan los límites superiores recomendados, en poblaciones donde la ingesta de calcio de la dieta ya es alta, probablemente no produzca mayores beneficios y tal vez tenga algún riesgo de eventos cardiovasculares.

Gabriela Cormick [ Instituto de efectividad Clínica y Sanitaria. gabmick@yahoo.co.uk ]

Cormick Gabriela. La relación entre la ingesta de calcio y los eventos cardiovasculares no es concluyente. Evid Act Pract Ambul. 2015;18(1)10 Ene-Mar. Comentado de: $\mathrm{Li} \mathrm{K}$, et al. Associations of dietary calcium intake and calcium supplementation with myocardial infarction and stroke risk and overall cardiovascular mortality in the Heidelberg cohort of the European Prospective Investigation into Cancer and Nutrition study. Heart. 2012;98(12):920-5. PMID: 22626900.

Referencias

1. ENNyS. (2007). Encuesta Nacional de Nutrición y Salud. Documento de resultados. Buenos Aires: Ministerio de Salud de la Nación.

2. Crichton GE. Dairy consumption and metabolic syndrome: a systematic review of findings and methodological issues. Obesity reviews $2011 ; 12(5): e 190-e 201$

3. Lewis JR, Zhu K, Prince RL. Adverse events from calcium supplementation: Relationship to errors in myocardial infarction self-reporting in randomized controlled trials of calcium supplementation. J Bone Miner Res. 2012 Mar;27(3):719-22.

4. Sabbagh Z, Vatanparast H. Is calcium supplementation a risk factor for cardiovascular diseases in older women? Nutr Rev. 2009 Feb; 67(2):105-8.

5. Hsia J, Heiss G, Ren H, et al.Calcium/vitamin D supplementation and cardiovascular events. Circulation. 2007 Feb 20;115(7):846-54.

6. Bolland MJ, et al. Effect of calcium supplements on risk of myocardial infarction and cardiovascular events: meta-analysis. BMJ. 2010 Jul 29;341:c3691.

7. Wang L, et al. Systematic review: Vitamin D and calcium supplementation in prevention of cardiovascular events. Ann Intern Med. 2010 Mar 2;152(5):315-23.

8. Hennekens $\mathrm{CH}$, Barice EJ. Calcium supplements and risk of myocardial infarction: a hypothesis formulated but not yet adequately tested. Am J Med. 2011 Dec;124(12):1097-8.

9. Reid IR, et al. Calcium supplements and risk of myocardial infarction: an hypothesis twice tested. Am J Med. 2012 Apr;125(4).

10. Daly RM. Ebeling PR. Is Excess Calcium Harmful to Health? Nutrients. 2010 May:2(5):505-22.

10 EVIDENCIA - Actualización en la Práctica Ambulatoria - Enero/Marzo 2015 - Disponible en internet: www.evidencia.org 